\title{
The abnormal right ventricle: Relevant on low risk SPECT perfusion images?
}

\author{
Richard C. Brunken, MD, FAHA, FACC, FASNC ${ }^{\mathrm{a}, \mathrm{b}}$ \\ a Department of Radiology, Cleveland Clinic Lerner College of Medicine of Case Western \\ Reserve University, Cleveland, $\mathrm{OH}$ \\ b Department of Nuclear Medicine/Jb3, Cleveland Clinic, Cleveland, $\mathrm{OH}$
}

Received Apr 13, 2021; accepted Apr 13, 2021

doi: $10.1007 / \mathrm{s} 12350-021-02647-1$

\section{See related article, pp. 1903-1914}

Assessment of right ventricular (RV) perfusion, function, and metabolism can provide additional diagnostic and prognostic information in patients with cardiovascular disorders. $^{1-8}$ Readers of SPECT myocardial perfusion images may anticipate abnormal RV dilatation or hypertrophy in some patients with pulmonary hypertension (PH), heart failure, valvular heart disease, and/or ischemic heart disease. However, the RV sometimes appears abnormal on SPECT images even when the left ventricle (LV) is entirely normal. In the absence of antecedent heart disease or PH the clinical significance of these observations is uncertain. Accordingly, Jose and colleagues sought to examine the ramifications of an abnormal $\mathrm{RV}$ on low-risk SPECT perfusion studies acquired over a thirteen- year period at the University of Cincinnati. ${ }^{9}$

\section{STUDY DESIGN}

They reviewed 12,883 SPECT perfusion records and identified 4,761 exams with normal LV perfusion. None of the study patients had heart failure, $\mathrm{PH}$, prior MI or valvular disease, and none demonstrated transient LV dilatation with stress. A standard report template required the RV to be classified normal or abnormal. Right ventricular hypertrophy (RVH) was considered present when the wall thickness was comparable to that

Reprint requests: Richard C. Brunken, MD, FAHA, FACC, FASNC, Department of Nuclear Medicine/Jb3, Cleveland Clinic, 9500 Euclid Avenue, Cleveland, OH 44195; rbrunken09@charter.net

J Nucl Cardiol 2022;29:1915-8.

1071-3581/\$34.00

Copyright (C) 2021 American Society of Nuclear Cardiology. of the LV, and tracer uptake was $50 \%$ or greater of the LV. Right ventricular dilatation (RVD) was defined by an RV appearing larger than the LV. RV findings were related to all-cause mortality, all-cause hospitalization and the onset of PH on echocardiography. (Patients with new PH were required to have a baseline echocardiogram prior to perfusion imaging to exclude pre-existing PH.) Demographic and clinical data were analyzed using statistical models adjusted for age, gender, race, body mass index, and for the comorbidities of diabetes, hypertension, chronic kidney disease (CKD), obstructive sleep apnea (OSA), and chronic obstructive pulmonary disease (COPD) as identified by ICD-10 codes in the medical record.

\section{RELATIONSHIP TO MORTALITY}

The authors report that the RV was abnormal in 410 (8.6\%) of the SPECT studies with normal LV perfusion, with 288 exhibiting RVH and 146 RVD. Over a median follow-up period of 95.5 months, there were 494 deaths $(10.4 \%)$, with a median time to death of 69.8 months. Of note, 2204 patients $(46.3 \%)$ did not have full vital status information at the time of data censoring. However, the investigators argue that patients lost to follow-up had clinical characteristics similar to those of the initial cohort (Supplemental Table S2) and that the loss of this information would be unlikely impact the study results.

RV abnormalities were independently associated with all-cause mortality (HR 1.41, CI 1.07-1.86, $P=.015)$. Nearly twice as many deaths occurred in those with an abnormal RV as compared to those with a normal RV $(18.5 \%$ vs $9.6 \%, P<.0001)$. In a multivariable Cox proportional hazards model, an RV abnormality had the highest hazard ratio for mortality. Further analysis revealed that the mortality risk was attributable to RVH (hazard ratio of $1.49, P=.011$ ) and not to RVD (hazard ratio of $1.01, P=.977$ ). 


\section{RELATIONSHIP TO NEW PH/HOSPITAL ADMISSION}

Nearly half of the study cohort, $45 \%$, did not have echocardiography after nuclear imaging. However, new echocardiographic evidence of $\mathrm{PH}$ was identified in 619 patients $(13.0 \%)$, with a median time to diagnosis of 37.6 months. Patients with an abnormal RV were more than twice as likely to develop PH $(25.9 \%$ vs $11.8 \%$, $P<.0001)$. In the authors' statistical model, an abnormal RV had the highest adjusted hazard ratio (2.06) for the development of PH. Both RVH (hazard ratio 1.90, $P<.001$ ) and RVD (hazard ratio $1.59, P=.016$ ) were associated with the development of $\mathrm{PH}$.

There were 2,487 hospital admissions in the study cohort (median time to admission was 27.7 months); 232 (56.6\%) with an abnormal RV were admitted compared to $2,255(51.8 \%)$ with a normal RV $(P=.07)$. Thus, the presence of an RV abnormality on SPECT images was not statistically associated with all-cause hospitalization. The authors suggested that this may reflect the fact that all admissions were considered, including those for unrelated medical or psychiatric disorders.

The authors conclude that " $\mathrm{RV}$ abnormalities found on low-risk SPECT imaging studies ... are independently associated with increased mortality and risk of developing echocardiographic $\mathrm{PH}$ and could identify highrisk patients for closer monitoring and additional diagnostic testing'".

\section{SOME CAUTIONARY NOTES}

Several factors should be considered regarding this study. A standard report template required the image reader to indicate if the RV was normal or abnormal, and to denote whether RVD or RVH was present. Although consistent collection of RV information is a study strength, there are concerns regarding the retrospective nature of the investigation. The Electronic Medical Record Search Engine (EMERSE) was used to "mine" the text of medical records associated with the SPECT images. As the authors acknowledge, text mining reflects the completeness and accuracy of the information in the clinical medical record. In a population with median BMI of $30 \mathrm{~kg} \mathrm{~m}^{-2}$ and age of 50 years, the prevalence of hypertension, diabetes/CKD, and COPD/ OSA was $5.8 \%, .2 \%$ and $.5 \%$, respectively. These values seem low relative to other reports. ${ }^{10-14}$ For example, the CDC National Diabetes Statistics Report of 2020 notes a prevalence of diagnosed diabetes in adult males of $11.0 \%$ and $9.5 \%$ in females,${ }^{10}$ roughly 50 times greater than in the study cohort. Similarly, prior reports indicate higher prevalences of hypertension, CKD and COPD/ OSA. This raises the concern that ICD-10 codes may have under-estimated the number of patients with comorbid medical conditions, which could potentially impact the study findings.

A second concern is the loss of follow-up vital status in nearly half of the patients. Although the clinical and demographic characteristics of those without follow-up are comparable to the original cohort, it is not possible to exclude the possibility that data loss may have skewed the results.

RV observations were not considered according to the type of image acquired. Images were obtained on two different cameras and some had attenuation correction while some did not. Findings on rest images were not distinguished from stress images, and exercise stress images were not distinguished from vasodilator stress images. Exercise and vasodilator stress may exert different physiologic effects on both LV and the RV. In this sense, the investigation would have benefitted from an "apples to apples" comparison stratifying the findings by rest/stress mode and camera of acquisition. Furthermore, the authors did not consider transient RV abnormalities (those present with stress but not rest). Given that Williams reported that transient RV dilation with stress may reflect balanced $\mathrm{CAD},{ }^{7}$ it is possible that identification of changes in RV size or tracer uptake with stress could have contributed further to the study findings.

Most of the SPECT images acquired over the thirteen-year study period were read by two physicians board certified in Nuclear Cardiology. In a small random sample (16 studies, equivalent to about $.3 \%$ of the image sets in the study cohort), the observers demonstrated good agreement on the presence/absence of an abnormal RV (93.8\%), RVH (100\%) and RVD (86.7\%). While encouraging, little additional information is available regarding the intra- and inter- observer reproducibility of all readers and whether the degree of observer agreement was invariant over the rather long time period of the investigation.

\section{WHAT ABOUT THE LV?}

Neither left ventricular systolic nor diastolic function was considered in this study. Presumably, LV function was considered normal in the subjects as perfusion was normal. However, modest differences in LV function between the original cohort and those lost to follow-up could have impacted the study findings. Furthermore, it is of interest that indices of diastolic LV function were not considered in the statistical model, as most patients (64\%) with the onset of catheterizationconfirmed PH had elevated pulmonary pressures due to left heart disease, as identified by elevated pulmonary capillary wedge pressures. Perhaps RVH on SPECT 
images is a marker of $L V$ diastolic dysfunction as an indicator of mortality risk, at least in some patients. As such, this observation would have differing clinical ramifications than $\mathrm{RVH}$ arising from purely pulmonary vascular sources.

\section{CHALLENGES FOR NUCLEAR CARDIOLOGY}

This report provides further impetus to examine the consequences of an abnormal RV on SPECT images. The initial challenge is to understand how the SPECT RV signal relates to actual ventricular volumes and masses, as measured by independent gold standards (e.g. phantom studies or MRI images). How do partial volume effect, photon attenuation, respiratory motion ${ }^{15}$ and image acquisition and reconstruction techniques impact RV counts and volumes? To what extent do abnormalities in the thoracic cavity impact the visualized $\mathrm{RV}^{16,17}$ ? Is an RV count density of $50 \%$ of $\mathrm{LV}$ really the best means to define RVH? Can prominent RV tracer uptake be observed in the absence of RVH, for example when there is a global reduction in $\mathrm{LV}$ counts ${ }^{18}$ ? What would be the ideal cut-off value for $\mathrm{RVH}$ if there is marked $\mathrm{LVH}$ with prominent tracer uptake? Alternatively, could some quantitative measure of absolute RV uptake, such as a SUV measurement on an ungated summed image provide a better way of identifying RVH?

Most SPECT readers will readily recognize severe RVD, but quantitative volume measurements could prove more accurate to identify lesser degrees of dilatation, and for identifying volume changes from rest to stress. Measurements of RV size, function and tracer uptake $^{19}$ could provide a more consistent means of identifying and characterizing the "abnormal" RV than the subjective impressions of even an experienced human observer. In short, we need to "calibrate" the signal abnormalities on SPECT images to actual RV volumes and masses, as defined by independent gold standards.

Once the RV signal has been calibrated, we need to reach a consensus on what is normal and mildly/moderately/severely abnormal. These findings then need to be consistently reported in a structured clinical document. ${ }^{20}$ ASNC guidelines note that "SPECT and PET MPI also allow interpretation of the perfusion, size and global and segmental function of the right ventricle', However, the document indicates that these descriptors are optional and not typically reported unless abnormal. ${ }^{20}$ Considering the study of Jose and colleagues, a structured nuclear cardiology report would benefit by inclusion of information about RV perfusion, measurements of RV tracer uptake (relative to LV, SUVs), stress and rest RV volumes, stress and rest RV ejection fractions, and global and regional RV function for each SPECT perfusion study. Once this data is then integrated with the appropriate patient demographic and clinical information, it will then enable us to definitively understand the significance of an abnormal RV on low risk SPECT perfusion images.

\section{References}

1. Sanz J, Sanchez-Quintana D, Bossone E, Harm J, Bogaard HJ, Naeije R. Anatomy, function, and dysfunction of the right ventricle: JACC state-of-the art review. J Am Coll Cardiol 2019;73:1463-82.

2. Mannting F, Zabrodina YV, Dass C. Significance of increased right ventricular uptake on 99mTc-sestamibi SPECT in patients with coronary artery disease. J Nucl Med 1999;40:889-94.

3. Kim J, Cho SG, Kang SR, Yoo SW, Kwon SY, Min JJ, et al. Association between FDG uptake in right ventricular myocardium and cancer-therapy induced cardiotoxicity. J Nucl Cardiol 2020;27:2154-63.

4. Tuominen H, Haarala A, Tikkakoski A, Kahonen M, Nikus K, Sipila K. FDG-PET in possible cardiac sarcoidosis: Right ventricular uptake and high total cardiac metabolic activity predict cardiovascular events. J Nucl Cardiol 2021;28:199-205.

5. Ahmadi A, Renaud JM, Promislow S, Burwash IG, Dwivedi G, Klein R, et al. Increased myocardial oxygen consumption rates are associated with maladaptive right ventricular modeling and decreased event-free survival in heart failure patients. J Nucl Cardiol 2020. https://doi.org/10.1007/s12350-020-02144-X.

6. Meyer P, Filippatos GS, Ahmed MI, Iskandrian AE, Bittner V, Perry GJ, et al. Effects of right ventricular ejection fraction on outcomes in chronic systolic heart failure. Circulation 2010;121:252-8.

7. Williams KA, Schneider CM. Increased stress right ventricular activity on dual isotope perfusion SPECT a sign of multivessel and/or left main coronary artery disease. J Am Coll Cardiol 1999;34:420-7.

8. Pueschner A, Chattranukulchai P, Heitner JF, et al. The prevalence, correlates, and impact on cardiac mortality of right ventricular dysfunction in nonischemic cardiomyopathy. J Am Coll Cardiol Img 2017;10:1225-36.

9. Jose A, Zhou C, Baker R, Walker J, Kurek N, O'Donnell RE, et al. Predictive value of incidental right ventricular abnormalities identified on SPECT for mortality and pulmonary hypertension. $\mathbf{J}$ Nucl Cardiol 2021. https://doi.org/10.1007/s12350-021-02612-y.

10. Centers for Disease Control and Prevention. National Diabetes Statistics Report, 2020. Atlanta, GA: Centers for Disease Control and Prevention, U.S. Dept of Health and Human Services 2020. https://www.cdc.gov/diabetes/pdfs/data/statistics/national-diabetes -statistics-report.pdf

11. National Center for Health Statistics. Health, United States, 2019: Table 21. Selected health conditions and risk factors, by age: United States, selected years 1988-1994 through 2017-2018. Hyattsville, MD 2021. https://www.cdc.gov/nchs/hus/content s2019.htm

12. Biener AI, Decker SL, Rohde F. Prevalence and treatment of chronic obstructive pulmonary disease (COPD) in the United States. JAMA 2019;322:602. https://doi.org/10.1001/jama.2019. 10241.

13. Vart P, Powe NR, McCulloch CE, Saran R, Gillespie BW, Sayda $\mathrm{S}$, for the Centers for Disease Control and Prevention Chronic Kidney Disease Surveillance Team. National trends in the 
prevalence of chronic kidney disease among racial/ethnic and socioeconomic status groups, 1988-2016. JAMA Netw Open 2020; 3: e207932. https://doi.org/10.1001/jamanetworkopen.2020.7932

14. Benjafield AV, Ayas NT, Eastwood PR, Heinzer R, Ip MSM, Morrell MJ, et al. Estimation of the global prevalence and burden of obstructive sleep apnoea: A literature-based analysis. Lancet Respir Med 2019;7:687-98.

15. Pretorius PH, Johnson KL, Dahlberg ST, King MA. Investigation of the physical effects of respiratory motion compensation in a large population of patients undergoing Tc- $99 \mathrm{~m}$ cardiac perfusion SPECT/CT stress imaging. J Nucl Cardiol 2020;27:80-95.

16. Akbulut A, Kalayci S, Aydinbelge FN, Koca G, Korkmaz M. Pneumonectomy-related small omega $(\omega)$ sign and increased pulmonary uptake in myocardial perfusion imaging. J Nucl Cardiol 2019;26:2154-8.

17. Kalayci S, Akbulut A, Koca G, Yigit H, Korkmaz M. Barrier effect of sternum to right ventricle dilatation appearing like letter phi “ $\varphi$ ”" on MPI. J Nucl Cardiol 2018;25:1433-5.
18. Vadi SK, Mehrotra S, Sood A, Parmar M, Dasagrandhi V, Kaur K, et al. Flip-flop right ventricle myocardial perfusion on stress-rest 99mTc-MIBI myocardial perfusion scintigraphy: An indirect evidence for severe left ventricular coronary arterial disease? J Nucl Cardiol 2019;26:1033-5.

19. Farag AA, Heo J, Tauxe L, Bhambhvani P, Germano G, Kavanagh $\mathrm{P}$, et al. Detection and quantitation of right ventricular reversible perfusion defects by stress SPECT myocardial perfusion imaging: A proof-of-principle-study. J Nucl Cardiol 2019;26:266-71.

20. Tilkemeier PL, Bourque J, Doukky R, Sanghani R, Weinberg RL. ASNC imaging guidelines for nuclear cardiology procedures Standardized reporting of nuclear cardiology procedures. J. Nucl. Cardiol 2017; 24: 2064-2128. https://doi.org/10.1007/s12350-0171057-y

Publisher's Note Springer Nature remains neutral with regard to jurisdictional claims in published maps and institutional affiliations. 